\title{
The preventive role of Spirulina platensis (Arthrospira platensis) in immune and oxidative insults in a stress-induced rat model
}

\author{
Nilay Seyidoğlu ${ }^{1 凶}$, Eda Köşeli ${ }^{2}$, Rovshan Gurbanlı ${ }^{2}$ Cenk Aydın² \\ ${ }^{1}$ Department of Physiology, Faculty of Veterinary Medicine, \\ Tekirdağ Namık Kemal University, 59030 Tekirdağ, Turkey \\ ${ }^{2}$ Department of Physiology, Faculty of Veterinary Medicine, \\ Bursa Uludağ University, 16059 Bursa, Turkey \\ nseyidoglu@nku.edu.tr
}

Received: October 23, 2020

Accepted: May 25, 2021

\begin{abstract}
Introduction: There is a balance between oxidative stress, antioxidant capacity and immune response. Their roles in physiological and behavioural mechanisms are important for the maintenance of the organism's internal equilibrium. This study aimed to evaluate the antioxidant effects of the exogenous alga Spirulina platensis (Arthrospira platensis) in a stress-induced rat model, and to describe its possible mechanism of action. Material and Methods: Thirty-six adult male Sprague Dawley rats were separated into four groups: control (C), stress (S), S. platensis (Sp), and S. platensis + stress (SpS). The rats in groups Sp and SpS were fed with $1,500 \mathrm{mg} / \mathrm{kg}$ b.w./day Spirulina platensis for 28 days. All rats were exposed to prolonged light phase conditions (18 h light: $6 \mathrm{~h}$ dark) for 14 days. The $\mathrm{SpS}$ and $\mathrm{S}$ groups were exposed to stress by being kept isolated and in a crowded environment. Blood samples were obtained by puncturing the heart on the 28th day. The effect of stress on serum corticosterone, oxidative stress markers (TOS, TAC, PON1, OSI) and immunological parameters (IL-2, IL-4, IFN- - ) were tested. Also, the brain, heart, intestines (duodenum, ileum, and colon), kidney, liver, spleen, and stomach of the rats were weighed. Results: Serum corticosterone levels were higher in the $\mathrm{S}$ group than in the $\mathrm{C}$ group, and significantly lower in the $\mathrm{SpS}$ group than in the $\mathrm{S}$ group. Mean total antioxidant capacity were lower in the S group than in the C group, and Spirulina reversed this change. Although not significantly different, IL-2 was lower in the $\mathrm{S}$ group than in the $\mathrm{C}$ group. However, in the $\mathrm{SpS}$ group, IL-2 increased due to Spirulina platensis mitigating effects of stress. Conclusion: Male rats fed a diet with Spirulina platensis could experience significantly milder physiological changes during stress, although stress patterns may be different. Exogenous antioxidant supplements merit further investigation in animals and humans where the endogenous defence mechanism against stress may not be sufficient.
\end{abstract}

Keywords: corticosterone, immune function, oxidant-antioxidant status, Spirulina (Arthrospira) platensis, stress.

\section{Introduction}

The stress response is a natural mammalian process begun by the stimulus of different situations such as isolation, crowded environments, different light/dark regimens, and varying durations of given intensities of lighting. Any discussion of the effects of several stressors on animal physiology encounters the problem of defining how animals cope with stress. The release of stress-response hormones and cytokines is only one facet of stress reactions' complexity. The modulation of receptors, circulation of hormone transport-binding proteins and infiltrating macrophages, and intercommunication of hormone secretion pituitary cells lead to the stress response and modify regulatory processes. Research has clarified the neurocircuitry underlying the effects of corticotropinreleasing hormone on the hypothalamic-pituitaryadrenocortical axis and sympathetic adreno-medullary system $(23,35)$. The activation of the sympathetic nervous system and adrenal function has been seen in signs exhibited by rats kept in social isolation, alone and in a crowded environment and is a finding reported in several studies $(12,16,23)$.

Reactive oxygen species (ROS) are produced by living organisms as a consequence of normal cellular metabolism and exposure to environmental oxidants such as microbial infections, extensive exercise, or pollutants and toxins such as ozone, pesticides, 
UV radiation and smoke. Large ROS accumulation is associated with adverse modifications of cellular components such as lipids, proteins, and DNA, and is a contributing cause of cancer, high blood pressure, neurological disorders, atherosclerosis, asthma, and diabetes. Both endogenous and exogenous defences are effective against oxidative stress, but the endogenous defence mechanism may not be sufficient to totally protect against the effects of ROS. Deleterious effects of oxidative stress arise from an imbalance between production of ROS and the capacity of the antioxidant mechanism. If the antioxidant mechanisms in an organism are insufficient, exogenous antioxidant supplements should be added to the diet for better health. Supplementation is a means of combatting oxidative stress both in humans and animals. The exogenous defence mechanism comes from the diet in the form of antioxidants, especially from natural antioxidants as phytochemicals in vegetables, fruits, flowers and traditional medicinal plants. The importance of natural antioxidants is being increasingly often investigated in oxidative-antioxidative balance and wellness because of consumer concern regarding the safety of synthetic antioxidants, and because of the low cost and high capacity for $\mathrm{H}+$ donation of natural antioxidants (15). Besides endogenous antioxidants, exogenous antioxidants are essential to mitigate the harm of oxidative stress, and they must obviously be consumed in amounts sufficient to deliver their intended effect. It was reported that there is a correlation between total antioxidant capacity and consumption of herbs (5). Total antioxidant capacity (TAC) denotes the total effect of all antioxidants in a studied organism and analogously, total oxidant status (TOS) does so regarding the effect of all oxidants. In studies investigating TAC and TOS, it is pertinent to also evaluate paraoxonase-1 (PON1), an antioxidant enzyme that is produced by the liver. Paraoxonase- 1 has a role in decreasing oxidative stress. It was explained that as inflammation progresses in the response of the organism to oxidative stress, the TOS and TAC values increase, and PON1 decreases $(4,29)$.

Cytokines are produced in reaction to physiological challenges which are expressed by white blood cell levels $(23,33)$. It was shown that cytokines have a wide range of effects on the organism, including fever, inflammation, and promotion of wound healing. Excessive or insufficient production of cytokines is related to the pathophysiology of a range of diseases and conditions, stress among them $(14,31)$. Stress is accompanied by altered production of inflammatory cytokines and neuropeptides in the central nervous system. Contradictory results have been indicated for the relationship between stress and cytokines such as interleukins 2, 4 and 6 (IL-2, IL-4, and IL-6), and interferon gamma (IFN- $\gamma$ ). Some researchers reported a decrease in IL-2 during stress (21), while others found an increase (6). It was also reported that IL-4 production could be increased, decreased or unchanged by stress $(6,25,38)$. Some studies conducted on rats and mice showed higher IL-6 levels under stress circumstances (3). In contrast to some of the findings for IL-2, IL-4, and IL-6, it was reported that stress suppressed the production of IFN-y (19).

Natural antioxidants and their products have vast potential to benefit health if introduced to human and animal nourishment. Understanding natural antioxidants in the context of oxidative stress, and translation of this knowledge into improvement of animal and human health are significant challenges. Spirulina platensis is the microalga the most frequently used as a supplement. It contains carotenoids, phycocyanin, xanthophylls and phycobilins, which have antioxidant activity $(32,37)$.

The purpose of this study was to assess the influence of a diet supplemented with Spirulina platensis on different stress patterns in a rat model. We also focused on the antioxidant capacity, immune function, and organ weight after stress response. We hope that this study will extend knowledge of the therapeutic potential of Spirulina platensis among functional

food studies.

\section{Material and Methods}

Animals. Thirty-six healthy male Sprague Dawley rats aged 10-12 weeks old with an average body weight of 200-250 g were used in this study. The animals were housed under standard laboratory conditions $\left(22 \pm 1^{\circ} \mathrm{C} ; 55 \pm 10 \%\right.$ humidity) in clear, plastic cages $(42 \mathrm{~cm} \times 21 \mathrm{~cm} \times 20 \mathrm{~cm})$ with stainless steel feed hoppers. Wood shavings were used as bedding material.

Rats were given ad libitum access to a commercial rodent diet (Korkuteli Yem Gida San. A.S., Antalya, Turkey) and tap water. The basal diet contained 2000-2500 kcal $/ \mathrm{kg}$ energy and the content of crude protein was $23.50 \%$, crude oil was $2.95 \%$, crude cellulose was $5.92 \%$ and ash was $6.36 \%$. The chemical composition and ingredients of diets included $1.35 \%$ lysine, $0.98 \%$ phosphorus, $0.43 \%$ methionine, $0.85 \%$ calcium and $0.05 \%$ sodium.

Spirulina platensis (Egert, Izmir, Turkey) doses were applied by orogastric gavage that was modified from that used in previous research $(34,38)$. The microalga supplement contained $65 \%$ protein, $18 \%$ carbohydrate, $7 \%$ oils (especially linolenic, linoleic and arachidonic acid) and minerals.

Experiment design. The experimental rats were divided into four groups of nine animals and studied through an experimental protocol which was maintained for 28 days. The experimental groups were as follows: control (C), stress (S), Spirulina platensis (Sp), and Spirulina platensis + stress $(\mathrm{SpS})$.

Experiment execution. The first week was the acclimation period. The second and third weeks were the application period of Spirulina platensis. The alga was given to the $\mathrm{Sp}$ and $\mathrm{SpS}$ groups by orogastric gavage $(1,500 \mathrm{mg} / \mathrm{kg} / \mathrm{day})$ at the same time each day during the light period. 
The $\mathrm{C}$ and $\mathrm{S}$ groups only received the vehicle (tap water, $1 \mathrm{cc} /$ day) by orogastric gavage for 28 days.

For the last two weeks, all animals experienced a light: dark cycle of $18 \mathrm{~h}: 6 \mathrm{~h}$ with changes at 7:00 and 01:00, modified from precedents in the literature $(13,27$, 34). In these weeks, besides the rats in the $\mathrm{S}$ and $\mathrm{SpS}$ groups being fed with Spirulina platensis, they were exposed to two episodes of chronic stress.

Isolation stress was applied during the third week of the study to the $\mathrm{S}$ and $\mathrm{SpS}$ groups. The rats were kept in a separate cage for $30 \mathrm{~min}$ on Monday, Wednesday, Friday, and Sunday. Neither food nor water was given to the rats during this stress application.

Crowded environment stress was applied during the fourth week of the study to the $\mathrm{S}$ and $\mathrm{SpS}$ groups by placing six rats in a cage of a size specified for only three for $30 \mathrm{~min}$ on Tuesday, Thursday, and Saturday. As was the case for the previous stress, neither food nor water was given to the rats during this stress application.

Measurements. Blood samples were obtained by puncturing the heart under short (2-3 min) isoflurane anaesthesia at the end of the study. The brain, heart, intestines (duodenum, ileum, and colon), kidney, liver, spleen, and stomach of the rats were weighed on a BL210S balance (Sartorius, Göttingen, Germany) immediately after the animals were sacrificed.

Blood samples were centrifuged on the same day to separate the serum, and then the serum samples were kept at $-80^{\circ} \mathrm{C}$ until the analysis. The serum TOS, TAC and PON1 values were measured with commercial kits (Rel Assay Diagnostics kits; Mega Tip, Gaziantep, Turkey) by using colorimetric methods and a UV2600-VIS spectrophotometer (Shimadzu, Kyoto, Japan).

Measurement of TOS. The serum TOS value was determined using a novel automated measurement method and kit (catalogue no RL0024; Rel Assay Diagnostics, Mega Tip, Gaziantep, Turkey) $(9,10)$. The oxidants in the sample oxidise the ferrous ion complex to ferric ions, which are coloured by chromogen (xylenol orange) in the acidic medium. The colour (and therefore the sample's oxidant molecule concentration) is measured spectrophotometrically. The assay is calibrated with hydrogen peroxide and its results are expressed in micromolar hydrogen peroxide equivalent per liter ( $\mu \mathrm{mol} \mathrm{H}_{2} \mathrm{O}_{2} \mathrm{Eq} / \mathrm{L}$ ) and finally calculated to $\mathrm{mmol} / \mathrm{L}$.

Measurement of TAC. Serum TAC was also determined using a novel automated measurement method and kit (catalogue no RL0017; Rel Assay Diagnostics, Mega Tip, Gaziantep, Turkey) $(9,10)$. In the assay, a hydroxyl radical, the most potent biological radical, is produced. The ferrous ion solution in reagent 1 is mixed with reagent 2 (hydroxyl peroxide). Thereby, the brown cation dianisidine is produced. The assay has precision values of $>97 \%$ and yields results expressed as mmol of Trolox Eq/L.

Measurement of PON1. The serum PON1 (catalogue no RL0031, Rel Assay Diagnostics kits, Mega Tip, Gaziantep, Turkey) was determined using a novel-automated measurement method $(9,10)$. The sample is mixed with reagent 1 , which contains calciumbuffered PON1 cofactor enzyme, and reagent 2, which is a stable substrate solution. The absorbance of p-nitrophenol produced from paraoxon is measured. Nonenzymatic hydrolysis of paraoxon is subtracted from the total hydrolysis ratio. The unit of $1 \mu \mathrm{mol}$ paraoxonase activity is hydrolysed per litre, and finally calculated to U/L.

Oxidative stress index. The oxidative stress index (OSI) was calculated as the ratio percentage of total oxidant status to antioxidant status. For the calculation, TAC and TOS values were converted to $\mathrm{mmol} / \mathrm{L}$ and calculated according to the following formula:

OSI $=$ TOS $(\mathrm{mmol} / \mathrm{L}) / \mathrm{TAC}(\mathrm{mmol} / \mathrm{L})(9)$.

Serum corticosterone (Elabscience, Houston, TX, USA), IL-2, IL-4 and IFN-y were measured using a commercially available rat ELISA kit (ThermoFisher Scientific, Waltham, MA, USA). Assays were performed colorimetrically using a microplate reader (Biotek, Epoch, USA).

ELISA method for IL-2, IL-4, and IFN-y. Serum was diluted $1: 2$ in $2 \%$ bovine serum albumin in phosphate buffered saline (PBS) containing $0.05 \%$ Tween 20 (PBST; Sigma-Aldrich, St. Louis, MO, USA) and $100 \mu \mathrm{L}$ was added to the wells. Biotin-conjugated (Sigma-Aldrich, St. Louis, MO USA) detection antibody was added and the solution was incubated for $2 \mathrm{~h}$ at $18-25^{\circ} \mathrm{C}$. Afterwards, the plates were washed three times in PBST and streptavidin-HRP was added. Then after $1 \mathrm{~h}$ incubation at $18-25^{\circ} \mathrm{C}$ with shaking, the microwells were again washed three times, tetramethylbenzidine (Thermo Fisher Scientific) was added, and the solution was incubated again for $10 \mathrm{~min}$ at $18^{\circ} \mathrm{C}$. The reactions were stopped by adding $0.9 \mathrm{M} \mathrm{H}_{2} \mathrm{SO}_{4}(100 \mu \mathrm{L})$ and the product absorbance was determined at $450 \mathrm{~nm}$. The non-specific background (measured at $630 \mathrm{~nm}$ ) was subtracted.

ELISA method for serum corticosterone. Serum was diluted $1: 2$ in $2 \%$ bovine serum albumin in PBST and $100 \mu \mathrm{L}$ was added to the wells. Following the addition of the biotin-conjugated detection, the plates were incubated for $45 \mathrm{~min}$ at $37^{\circ} \mathrm{C}$, and then washed three times in PBST. Afterwards, streptavidin-HRP was added and the plates were incubated for $30 \mathrm{~min}$ at $37^{\circ} \mathrm{C}$. The microwells were washed five times, substrate reagent was added, and the solution was incubated again for $15 \mathrm{~min}$ at $37^{\circ} \mathrm{C}$. The reactions were stopped by the addition of $50 \mu \mathrm{L}$ of acid solution (E-EL-003096T, Elabscience) and the product absorbance was determined at $450 \mathrm{~nm}$.

Statistical assessment. Statistical analyses were performed with SPSS Version 17.0 (SPSS, Chicago. IL, USA). Data were tested for normality distribution and variance homogeneity assumptions. All the values were grouped and means and standard errors were calculated. One-way ANOVA was completed for all parameters to examine the difference between groups. Differences were considered significant at $\mathrm{P}<0.05$. If the difference between groups was significant $(\mathrm{P}<0.05)$, then a Tukey's test was completed to evaluate the differences. 
In non-homogenous groups, differences between means were analysed by the Kruskal-Wallis method and proved by a Mann-Whitney U test between the groups one by one.

\section{Results}

Our results showed that the levels of serum corticosterone significantly increased in the $\mathrm{S}$ group when compared to the $\mathrm{C}$ group (Fig. 1A; $46.02 \pm 1.72 \mathrm{ng} / \mathrm{mL}$ and $57.03 \pm 1.84 \mathrm{ng} / \mathrm{mL}$, respectively, indicating a $24 \%$ rise). This indicates stress was induced successfully. However, feeding with Spirulina platensis greatly reduced the upward change in corticosterone, as indicated by the $19 \%$ lower level in the $\mathrm{SpS}$ group compared to the $\mathrm{S}$ group.

A significant decrease in the mean TAC levels was observed in stress-induced and unsupplemented rats compared with those in control animals (Fig. 1B). Microalga-supplemented rats showed remarkable improvements in TAC levels as compared to stressinduced rats fed only the basal diet. However, there were no statistical differences found in TOS (Fig. 1C), PON1 (Fig. 1D) or OSI (Fig. 1E) values among the groups.

The IL-2, IL-4, and IFN- $\gamma$ parameters and their percentages are shown in Fig. 2. Although not significantly different when compared to control rats, IL-2 and IFN-y were lower in stress-induced animals. Feeding with Spirulina platensis reversed the IL-2 of rats exposed to stress. Nevertheless, there was a decline from the C group IL-4 levels in the S group, although not a significant one. In the study, there were no statistical differences in organ weights between groups, as shown in Table 1.
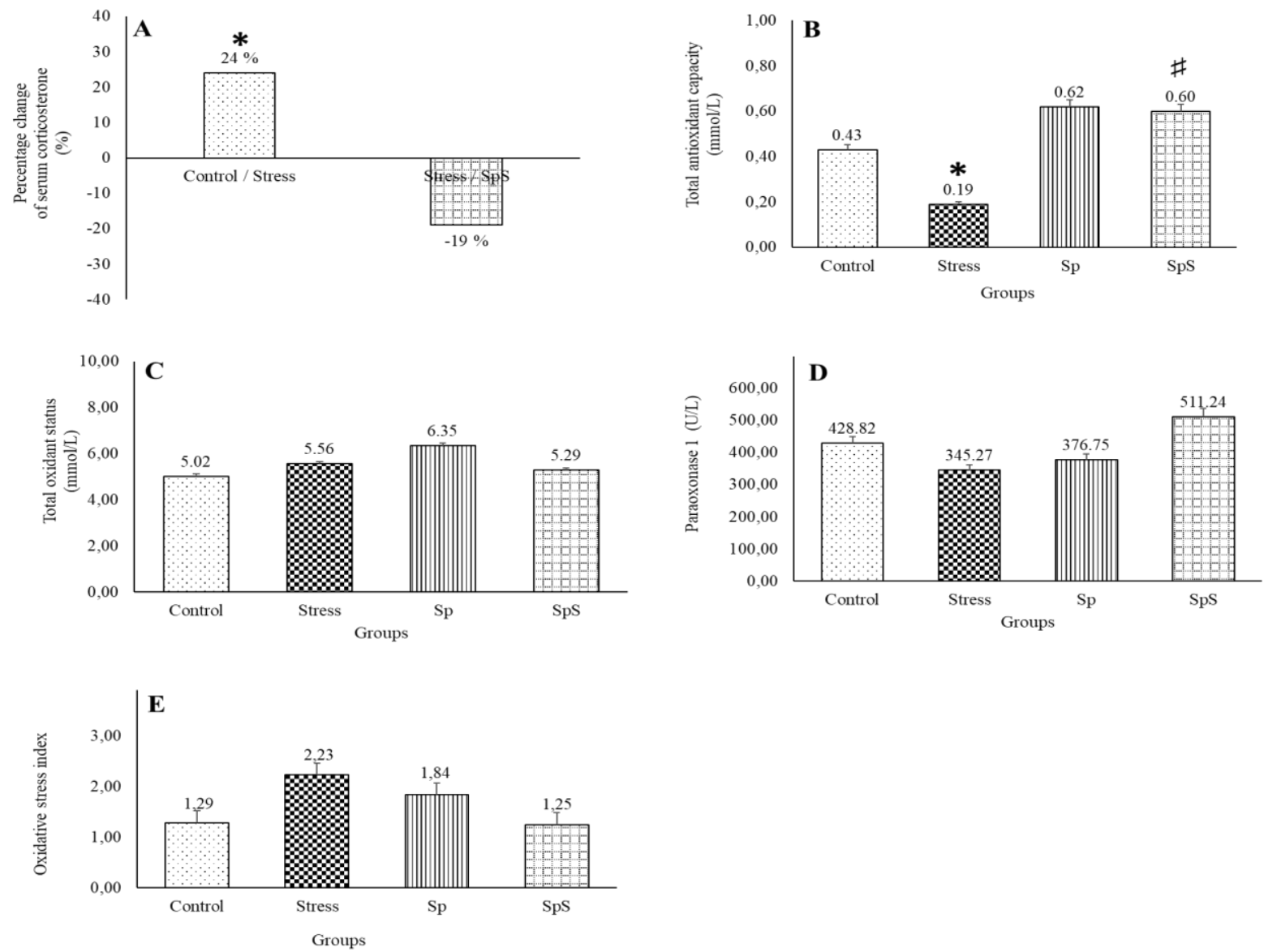

Groups: $\square$ Control, $\mathbb{\text { Stress, }} \square$ Sp: Spirulina platensis, 四 SpS: Spirulina platensis + stress

Fig. 1. Percentage change in the parameters indicative of stress response in the groups used for the study. A - mean serum corticosterone level: control versus stress groups and stress + Spirulina platensis versus stress groups; B - effect of supplementation with Spirulina platensis for 28 days on serum total antioxidant capacity; $\mathrm{C}$ - total oxidant status; $\mathrm{D}$ - paraoxonase 1 in stress-induced rats; $\mathrm{E}$ - oxidative stress index in stress-induced rats

All data are presented as the mean $\pm \mathrm{SD}(\mathrm{n}=9) ;{ }^{*}-\mathrm{P}<0.05$ when compared with control rat values; \# $-\mathrm{P}<0.05$ when compared with stressinduced rat values 

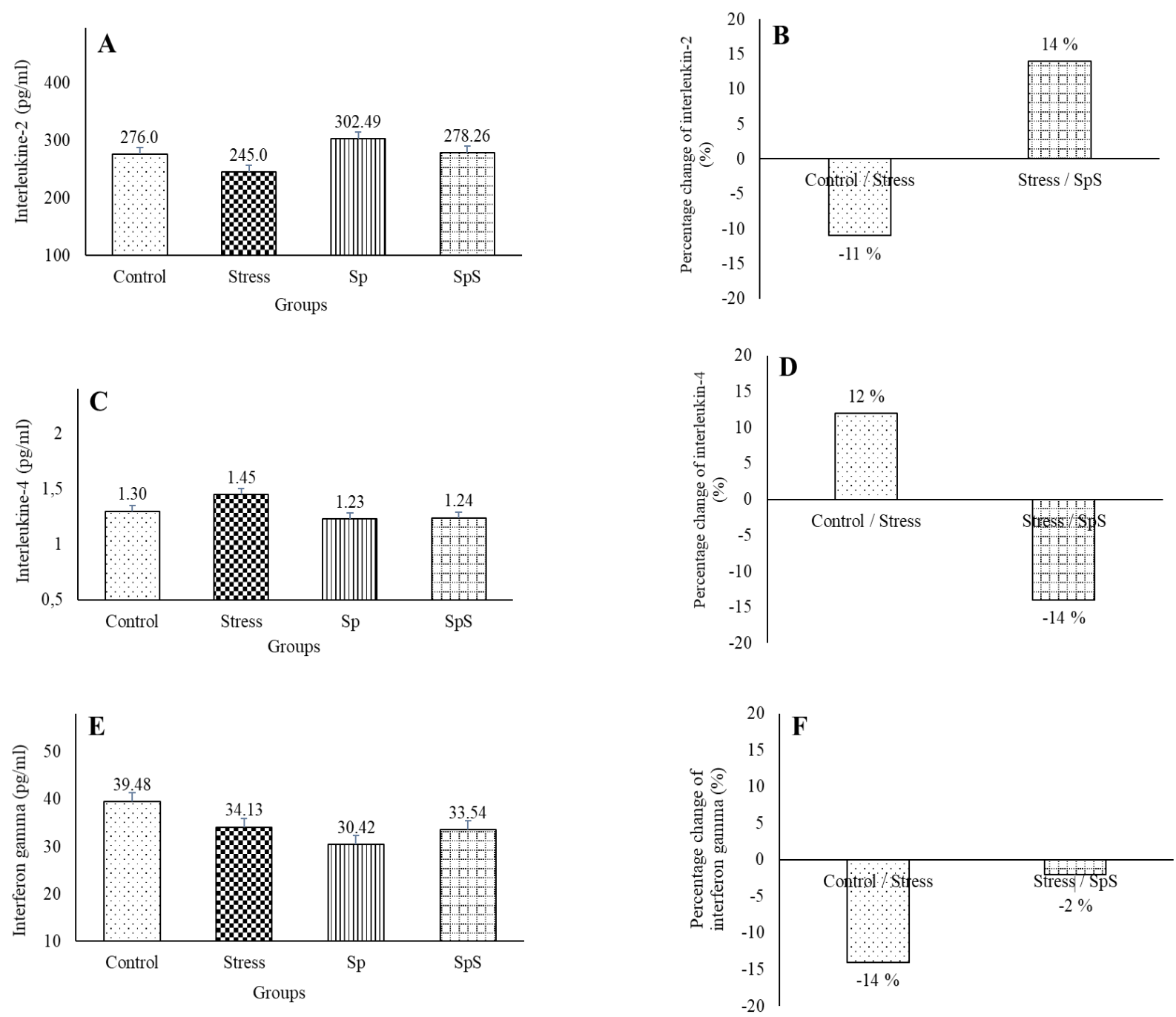

Groups: $\square$ Control, $\mathbb{0}$ Stress, $\square$ Sp: Spirulina platensis, $\square$ SpS: Spirulina platensis + stress

Fig. 2. Effect of supplementation with Spirulina platensis for 28 days on serum concentrations and percentage change in mean serum of interleukin 2, interleukin 4 and interferon gamma. A - interleukin 2 concentration; B - interleukin 2 percentage difference; $\mathrm{C}$ - interleukin 4 concentration; $\mathrm{D}$ - interleukin 4 percentage difference; $\mathrm{E}$ - interferon gamma concentration; $\mathrm{F}$ - interferon gamma percentage difference

All data are presented as the mean $\pm \mathrm{SD}(\mathrm{n}=9)$

Table 1. Effect of supplementation with Spirulina platensis for 28 days on organ weights in stressed rats All data are presented as the mean $\pm \operatorname{SD}(\mathrm{n}=9)$

\begin{tabular}{lcccc}
\hline \multirow{2}{*}{ Organ weights $(\mathrm{g})$} & \multicolumn{3}{c}{ Groups } \\
\cline { 2 - 5 } & Control $(\mathrm{C})$ & Stress $(\mathrm{S})$ & S. platensis $(\mathrm{Sp})$ & S. platensis + Stress $(\mathrm{SpS})$ \\
\hline Brain & $1.76 \pm 0.06$ & $1.75 \pm 0.13$ & $1.71 \pm 0.19$ & $1.73 \pm 0.10$ \\
Heart & $1.05 \pm 0.05$ & $1.03 \pm 0.09$ & $1.02 \pm 0.08$ & $1.03 \pm 0.14$ \\
Intestines & $25.15 \pm 2.25$ & $24.21 \pm 3.36$ & $25.84 \pm 1.00$ & $25.18 \pm 2.35$ \\
Kidney & $2.63 \pm 0.17$ & $2.58 \pm 0.33$ & $2.67 \pm 0.11$ & $2.71 \pm 0.43$ \\
Liver & $12.09 \pm 1.42$ & $11.93 \pm 1.34$ & $11.94 \pm 0.67$ & $11.89 \pm 1.63$ \\
Spleen & $0.67 \pm 0.07$ & $0.66 \pm 0.03$ & $0.65 \pm 0.05$ & $0.67 \pm 0.03$ \\
Stomach & $4.04 \pm 1.38$ & $3.95 \pm 0.70$ & $5.18 \pm 1.01$ & $4.93 \pm 0.83$ \\
\hline
\end{tabular}

\section{Discussion}

In this study, we evaluated the association between feeding male rats with Spirulina platensis and stress response-induced changes in mixed stress models. We provide evidence that 28 days of feeding with Spirulina platensis showed a protective effect during different stress conditions, which was manifested in serum corticosterone levels and total antioxidant capacity.

Stress responses have been linked to physiological conditions: besides the aforementioned serum corticosterone levels, they have been correlated with oxidative stress/antioxidant balance and cytokine levels. Serum corticosterone levels were significantly higher after 
induced mixed stress. Similar results were observed by Jameel et al. (18) who observed that corticosterone levels were increased when rats were subjected to forced swim and restraint tests. The administration of dietary Spirulina platensis resulted in significant decreases in serum corticosterone levels in rats. Another study focused on whether Spirulina platensis reduced serum corticosterone and glucose levels in experimentallyinduced stress (7). The authors concluded that consumption of the microalga reduced expression of some genes involved in stress response and showed beneficial effects on capacity for stress resistance in aquaculture practices. Also, dietary inclusion of $10 \%$ Spirulina platensis improved haematological and serum biochemical parameters of rainbow trout (Oncorhynchus mykiss) and can be cited as an immunostimulant technique (39).

Oxidative stress is a result of an increased generation of free radicals and/or reduced physiological activity of antioxidant defence against free radicals. Regarding the influence of Spirulina platensis on oxidative stress, we observed that supplementation with this antioxidant increased the serum concentration of TAC in the SpS group. This is probably associated with a positive correlation between the intake of Spirulina platensis and antioxidant activity (29). Results from experimental studies confirm the protective effects of blue-green alga against oxidative damage in vivo and in vitro. In New Zealand, in white rabbits with hypercholesterolemia, 5\% supplementation for eight weeks inhibited lipid peroxidation while increasing glutathione and the activities of glutathione peroxidase, glutathione reductase and glutathione transferase compared with the peroxidation intensity and activities of these antioxidants in rabbits maintained on unsupplemented feed. The protective effect of Spirulina platensis against oxidative stress has been largely attributed to its high content of phycocyanin (30). Studies in humans have confirmed that this alga confers protection against oxidative stress. Spirulina platensis supplementation of $8 \mathrm{~g}$ /day for 16 weeks given to healthy elderly Korean subjects showed an increase in the TAC status and levels of antioxidant enzymes (26). Daily intake of Spirulina platensis may mitigate oxidative stress and is associated with improved antioxidant capacity compared to experimental subjects not consuming the alga. PON1 is an antioxidant enzyme produced by the liver and regulating the metabolism in oxidative stress (4). During stress, while total oxidant capacity increases, total antioxidant capacity and paraoxonase value decrease. In the present study, although there was no statistical difference, the PON1 value decreased in the $S$ group compared to the $\mathrm{C}$ group, and increased in the $\mathrm{SpS}$ group compared to the $S$ group. These results may be related to antioxidant activity of Spirulina platensis counteracting oxidative imbalance.

Spirulina platensis has a powerful stimulating effect on the immune system by increasing the phagocytic activity of macrophages, causing natural killer cells to accumulate in tissues thereby stimulating the production of antibodies and cytokines and activating and mobilising $\mathrm{T}$ and $\mathrm{B}$ cells (11). This antioxidant also exerts a variety of immunomodulatory and anti-inflammatory effects by regulating key cytokines, including IL-1 $\beta$, IL-2, IL-4, IL-6, IL-10, and tumour necrosis factor alpha (8). We tested the effects of isolation and crowded environment stress on IL-2, IL-4 and IFN- $\gamma$ serum levels in male rats, and statistical analysis showed that the IL-2 and IFN-y serum levels decreased while the IL-4 level increased compared to non-stressed rats but that the differences were not statistically significant. Stress has been revealed as simultaneously enhancing and suppressing the immune response by altering the patterns of cytokine secretion $(24,33)$. Spirulina platensis can activate the management of antibodies and up- or downregulate the expression of cytokines encoding genes. Himmerich et al. (17) reported an increase in IL-2 and IL-4 and a decrease in IFN-y in rats under the two stress factors of forced swimming and repeated restraint. Researchers variously indicated that IL-4 decreased (22), increased (1) or remained unchanged (28) in acute and chronic stress models. These differences may be due to the presence of other active compounds in the microalga, such as vitamins, minerals, beta glucan, and phycocyanin.

Stress situations such as crowded environments or small cage sizes are also consequential for physical activity, feeding, and growth. It was reported that crowded environment stress suppressed rats' organ development and thereby their growth (2). This suppression is also explained by basal metabolism and hormonal profiles (40). In the present study, although no significant changes were observed, there was a decrease in some organ weights (brain, heart, intestines, kidney, liver, spleen, and stomach) in the $\mathrm{S}$ group compared to the $\mathrm{C}$ group.

The constant rise in the global human population threatens biodiversity and worsens air and water pollution. These negative consequences impact animal and human health in undesirable ways and disrupt homeostasis, and in this aspect may be regarded as stressors. Physicochemical stress can be caused by environmental agents and is manifested as chronic infections, autoimmune diseases, and other physiological disorders. Besides the aforementioned ramifications of environmental changes as threats to homeostasis, additional threats to it are several easily transmittable zoonoses. Because of these pathogenic risks, an organism's capability to maintain homeostasis should be assured by provision of exogenous natural antioxidants. People have been converted to phytochemicals as prophylaxis and therefore Spirulina platensis is readily accepted. We attempted to explain the relationship between mixed stress, oxidative-antioxidative balance and use of Spirulina platensis supplementation by evaluating the association between natural antioxidants and state of health. It can be suggested that the therapeutic potential 
of Spirulina platensis could act counter to the pathological changes occurring during mixed stress.

Conflict of Interest Statement: The authors declare that there is no conflict of interests regarding the publication of this article.

Financial Disclosure Statement: The source of funding of research and the article was the authors' own resources.

Animal Rights Statement: The experimental protocols were in accordance with the National Institute of Health Guide for the Care and Use of Laboratory Animals, and approved by the Animal Care and Use Committee of the University. The study was carried out with the permission of the Bursa Uludağ University Animal Experimentation Local Ethics Committee (approval no. 2018-06/08).

Acknowledgement: Special thanks to Prof. Dr. Mehmet Cansev for allocating his animal study room in the Experimental Animals Unit to us during research.

\section{References}

1. Almeida-Reis R., Toledo A.C., Reis F.G., Marques R.H., Prado C.M., Dolhnikoff M., Martins M.A., Leick-Maldonado E.A., Tibério I.F.L.C.: Repeated stress reduces mucociliary clearance in animals with chronic allergic airway inflammation. Respir Physiol Neurobiol 2010, 173, 79-85, doi: 10.1016/j.resp.2010.06.011.

2. Armario A., Ortiz R., Balasch J.: Effect of crowding on some physiological and behavioral variables in adult male rats. Physiol Behav 1984, 32, 35-37, doi: 10.1016/0031-9384(84)90066-0.

3. Bernberg E., Ulleryd M.A., Johansson M.E., Bergström G.M.: Social disruption stress increases IL-6 levels and accelerates atherosclerosis in $\mathrm{ApoE}^{-/-}$mice. Atherosclerosis 2012, 221, 359-365, doi: 10.1016/j.atherosclerosis.2011.11.041.

4. Camps J., Marsillach J., Joven J.: Measurement of serum paraoxonase-1 activity in the evaluation of liver function. World J Gastroenterol 2009, 15, 1929-1933, doi: 10.3748/wjg.15.1929.

5. Carlsen M.H., Halvorsen B.L., Holte K., Bøhn S.K., Dragland S., Sampson L., Willey C., Senoo H., Umezono Y., Sanada C., Barikmo I., Berhe N., Willet W.C., Phillips K.M., Jacobs D.R. Jr., Blomhoff R.: The total antioxidant content of more than 3100 foods, beverages, spices, herbs and supplements used worldwide. Nutr J 2010, 9, 3, doi: 10.1186/1475-2891-9-3.

6. Chuian O.M., Temur'iants N.A., Makhonina M.M., Zaiachnikova T.V.: Effect of hypokinetic stress and low intensity electromagnetic field of extremely high frequency on changes of cytokine concentration in rat blood [in Ukrainian]. Fiziol Zhurnal 2005, 51, 70-78.

7. De Mattos B.O., López-Olmeda J.F., Guerra-Santos B., Ruiz C.E., García-Beltrán J.M., Ángeles-Esteban M. Sánchez-Vázquez F.J., Fortes-Silva R.: Coping with exposure to hypoxia: modifications in stress parameters in gilthead seabream (Sparus aurata) fed spirulina (Arthrospira platensis) and brewer's yeast (Saccharomyces cerevisiae). Fish Physiol Biochem 2019, 45, 1801-1812, doi: 10.1007/s10695-019-00677-8.

8. El Sheikh S.M., Shalaby M.A.M., Hafez R.A., Metwally W.S.A., El-Ayoty Y.M.: The immunomodulatory effects of probiotic bacteria on peripheral blood mononuclear cells (PBMCS) of allergic patients. Am J Immunol 2014, 10, 116-130, doi: 10.3844/ajisp.2014.116.130.
9. Erel O.: A novel automated direct measurement method for total antioxidant capacity using a new generation, more stable ABTS radical cation. Clin Biochem 2004, 37, 277-285, doi: 10.1016/j.clinbiochem.2003.11.015.

10. Erel O.: A new automated colorimetric method for measuring total oxidant status. Clin Biochem 2005, 38, 1103-1111, doi: 10.1016/j.clinbiochem.2005.08.008.

11. Gad A.S., Khadrawy Y.A., El-Nekeety A.A., Mohamed S.R., Hassan N.S., Abdel-Wahhab M.A.: Antioxidant activity and hepatoprotective effects of whey protein and Spirulina in rats. Nutrition 2011, 27, 582-589, doi: 10.1016/j.nut.2010.04.002.

12. Gamallo A., Villanua A., Beato M.J.: Body weight gain and food intake alterations in crowd-reared rats. Physiol Behav 1986, 36, 835-837, doi: 10.1016/0031-9384(86)90439-7.

13. Gancarczyk M., Paziewska-Hejmej A., Carreau S., Tabarowski Z., Bilińska B.: Dose- and photoperiod-dependent effects of 17betaestradiol and the anti-estrogen ICI 182,780 on testicular structure, acceleration of spermatogenesis, and aromatase immunoexpression in immature bank voles. Acta Histochem 2004, 106, 269-278, doi: 10.1016/j.acthis.2004.04.002.

14. Gulati K., Guhathakurta S., Joshi J., Rai N., Ray A.: Cytokines and their Role in Health and Disease: A Brief Overview. MOJ Immunol 2016, 4, 00121, doi: 10.15406/moji.2016.04.00121.

15. Hall C. $3^{\text {rd }}$ : Chapter 9, Sources of natural antioxidants: oilseeds, nuts, cereals, legumes, animal products and microbial sources. In: Antioxidants in food: Practical applications, edited by J. Pokorny, N. Yanishlieva, M. Gordon, Woodhead Publishing, Cambridge, 2001, pp. 159-209, doi: 10.1016/9781855736160.3.159.

16. Hawkley L.C., Cole S.W., Capitanio J.P., Norman G.J., Cacioppo J.T.: Effects of Social Isolation on Glucocorticoid Regulation in Social Mammals. Horm Behav 2012, 62, 314-323, doi: 10.1016/j.yhbeh. 2012.05.011.

17. Himmerich H., Fischer J., Bauer K., Kirkby K.C., Sack U., Krügel, U.: Stress-induced cytokine changes in rats. Eur Cytokine Netw 2013, 24, 97-103, doi: 10.1684/ecn.2013.0338.

18. Jameel M.K., Joshi A.R., Dawane J., Padwal M., Joshi A., Pandit V.A., Melinkeri R.: Effect of various physical stress models on serum cortisol level in Wistar rats. J Clin Diagn Res 2014, 8, 181-183, doi: 10.7860/JCDR/2014/7210.4116.

19. Kour K., Sharma N., Chandan B.K., Koul S., Sangwan P.L., Bani S.: Protective effect of Labisia pumila on stress-induced behavioral, biochemical, and immunological alterations. Planta Med 2010, 76, 1497-1505, doi: 10.1055/s-0029-1240953.

20. Lee D.Y., Kim E., Choi M.H.: Technical and clinical aspects of cortisol as a biochemical marker of chronic stress. BMB Rep 2015, 48, 209-216, doi: 10.5483/bmbrep.2015.48.4.275.

21. Liu Y.L., Bi H., Fan R., Li Y.H., Wang Y.M., Chen Y.M., Chen J.Y., Chi S.-M., Pei J.-M.: Effect of compound nutrients on acute immobilization and cold water-immersion stress-induced changes of Th1/Th2 cytokines (in Chinese). Chin J Cell Mol Immunol $2012,28,601-603$

22. Lu Y., Liu M., Shi S., Jiang H., Yang L., Liu X., Zhang Q., Pan F.: Effects of stress in early life on immune functions in rats with asthma and the effects of music therapy. J Asthma 2010, 47, 526-531, doi: 10.3109/02770901003801964.

23. Marin M.T., Cruz F.C., Planeta C.S.: Chronic restraint or variable stresses differently affect the behavior, corticosterone secretion and body weight in rats. Physiol Behav 2007, 90, 29-35, doi: 10.1016/j.physbeh.2006.08.021.

24. Marshall G.D. Jr., Agarwal S.K., Lloyd C., Cohen L., Henninger E.M., Morris G.J.: Cytokine dysregulation associated with exam stress in healthy medical students. Brain Behav Immun 1998, 12, 297-307, doi: 10.1006/brbi.1998.0537.

25. Murakami M., Sato N., Sato N., Nakamura T., Masunaga H.: Changes in lymphocyte phenotypes and cytokine production by surgical stress in a rat small intestinal resection model. J Clin Biochem Nutr 2007, 40, 216-220, doi: 10.3164/jcbn.40.216.

26. Park H.J., Lee Y.J., Ryu H.K., Kim M.H., Chung H.W., Kim W.Y. A randomized double-blind, placebo-controlled study to establish the effects of Spirulina in elderly Koreans. Ann Nutr Metab 2008, 52, 322-328, doi: 10.1159/000151486. 
27. Park S.H., Kim I.-G., Kim H.-C., Gang M.-J.: Influence of various photoperiods on stress hormone production, immune function, and hematological parameters in ICR mice. Korean J Vet Res 2015 , 55, 111-116, doi: 10.14405/kjvr.2015.55.2.111.

28. Pertsov S.S., Koplik E.V., Stepanyuk V.L., Simbirtsev A.S.: Blood cytokines in rats with various behavioral characteristics during emotional stress and treatment with interleukin-1 $\beta$. Bull Exp Biol Med 2009, 148, 196, doi: 10.1007/s10517-009-0668-y.

29. Poljsak B., Milisav I.: The Neglected Significance of “Antioxidative Stress”. Oxid Med Cell Longev 2012, 2012, 480895, doi: 10.1155/2012/480895.

30. Romay Ch., González R., Ledón N., Remirez D., Rimbau V.: C-phycocyanin: a biliprotein with antioxidant, anti-inflammatory and neuroprotective effects. Curr Protein Pept Sci 2003, 4, 207-216, doi: 10.2174/1389203033487216.

31. Segerstrom S.C., Miller G.E.: Psychological stress and the human immune system: a meta-analytic study of 30 years of inquiry. Psychol Bull, 2004 130, 601-630, doi: 10.1037/00332909.130.4.601.

32. Seyidoğlu N., Aydin C.: Chapter 7, Stress, Natural Antioxidants and Future Perspectives. In: The Health Benefits of Foods Current Knowledge and Further Development, edited by C.L. Salanță, Intech Open, London, 2020, pp. 149-165, doi: 10.5772/intechopen.91167.

33. Sordillo L.M., Aitken S.L.: Impact of oxidative stress on the health and immune function of dairy cattle. Vet Immunol
Immunopathol 2009, 128, 104-109, doi: 10.1016/j.vetimm. 2008.10.305

34. Ten Hoor F., Rietveld W.J., Kooij M., Flory W.: Growth and 24 hour eating patterns of rats kept under various light:dark conditions. Lab Anim 1980, 14, 251-252.

35. Tsigos C., Chrousos G.P.: Hypothalamic-pituitary-adrenal axis, neuroendocrine factors and stress. J Psychosom Res 2002, 53, 865-871, doi: 10.1016/S0022-3999(02)00429-4.

36. Turner P.V., Vaughn E., Sunohara-Neilson J., Ovari J., Leri F. Oral Gavage in Rats: Animal Welfare Evaluation. J Am Assoc Lab Anim Sci 2012, 51, 25-30.

37. Wu L.-C., Ho J.A., Shieh M.-C., Lu I.W.: Antioxidant and antiproliferative activities of Spirulina and Chlorella water extracts. J Agric Food Chem 2005, 53, 4207-4212, doi: 10.1021/jf0479517.

38. Yang P.C., Jury J., Soderholm J.D., Sherman P.M., McKay D.M., Perdue M.H.: Chronic psychological stress in rats induces intestinal sensitization to luminal antigens. Am J Pathol 2006, 168, 104-114, doi: 10.2353/ajpath.2006.050575.

39. Yeganeh S., Teimouri M., Amirkolaie A.K.: Dietary effects of Spirulina platensis on hematological and serum biochemical parameters of rainbow trout (Oncorhynchus mykiss). Res Vet Sci 2015, 101, 84-88, doi: 10.1016/j.rvsc.2015.06.002.

40. Yıldız A., Hayırlı A., Okumus Z., Kaynar Ö. Kisa F. Physiological profile of juvenile rats: Effects of cage size and cage density. Lab Anim (NY) 2007, 36, 28-38, doi: 10.1038/ laban0207-28. 